\title{
Incidence of and Risk Factors for Postoperative Hyphema After 23-Gauge Pars Plana Vitrectomy for Proliferative Diabetic Retinopathy
}

\author{
Yu-Hong Nie \\ Yu Zhang \\ Fan Li \\ Qiong Wang \\ A-Min Xu \\ Zhen Chen
}

Department of Ophthalmology, Renmin Hospital of Wuhan University, Wuhan, 430060, People's Republic of China
Correspondence: Zhen Chen Department of Ophthalmology, Renmin Hospital of Wuhan University, No. 238, Wuchang Jiefang Road, Wuhan, 430060, People's Republic of China Tel +86 I387I025806

Email zhenchendr_09@I26.com
Purpose: We aim to investigate the incidence, clinical course, and risk factors for developing postoperative hyphema after 23-gauge pars plana vitrectomy (PPV) for patients with proliferative diabetic retinopathy (PDR) without neovascularization of the iris or angles and neovascular glaucoma (NVG).

Methods: This retrospective study included 155 consecutive eyes from 124 patients with at least three-month follow-up who underwent PPV for PDR without neovascularization of the iris or angles and NVG. Demographic data, surgery notes, postoperative hyphema assessment, intraocular pressure (IOP), and the surgical outcome were recorded.

Results: Postoperative hyphema occurred in 18 of 155 eyes (11.6\%), with 14 of those having hyphema on day 1 , and 4 having hyphema on days 7-20. Of the 18 eyes, only 3 (16.7\%) had normal IOP, and immediate intraocular hypertension was observed in 15 $(83.3 \%)$. Seven eyes required anterior chamber paracentesis and five needed anterior chamber irrigation. The average time for absorption of the hyphema was 13.1 days, and IOP was controlled in all cases. There was a significant correlation between membrane removal and the development of hyphema (OR $=5.65$ and 95\% CI: $1.190-25.203 ; p=0.013)$. No recurrence of hyphema was observed. In patients with hyphema, the final best corrected visual acuity (BCVA) was $1.75 \pm 0.84 \log$ MAR, which improved significantly compared to the initial BCVA of $2.20 \pm 0.65 \log$ MAR $(\mathrm{t}=3.893 ; \mathrm{p}=0.001)$, and the final anatomic success rate was $100 \%$.

Conclusion: The development of hyphema is not uncommon after PPV for patients with PDR without neovascularization of the iris or angles and NVG, and membrane removal is a risk factor for postoperative hyphema. The timely management of hyphema ensures that hyphema does not affect the visual recovery or the final anatomical success.

Keywords: pars plana vitrectomy, hyphema, proliferative diabetic retinopathy, diabetes, membrane removal

\section{Introduction}

Hyphema is defined as the accumulation of blood cells in the anterior chamber of the eye. ${ }^{1}$ The most common cause is ocular trauma (blunt or penetrating); ${ }^{2}$ however, it can also develop as a result of non-trauma-related conditions, such as iris neovascularization, melanoma, juvenile xanthogranuloma, retinoblastoma, leukemia, and medication-induced anticoagulation. ${ }^{3}$ In addition, hyphema can develop after ocular surgery, including surgeries for glaucoma, ${ }^{4-6}$ severe infectious keratitis, ${ }^{7}$ cataract, ${ }^{8,9}$ macular hole, ${ }^{10}$ epiretinal membrane, ${ }^{11}$ and rhegmatogenous 
retinal detachment. ${ }^{12}$ Hemorrhage in the anterior chamber blocks the angle of the chamber, inhibiting aqueous drainage and resulting in an increase in intraocular pressure (IOP). ${ }^{1,3}$ Hyphema is a common condition, and its prognosis is good if it is treated properly and timeously. However, hyphema can significantly alter a patient's visual prognosis through severe complications, such as corneal blood staining, amblyopia, secondary glaucoma, and optic atrophy. ${ }^{13}$ To our knowledge, postoperative hyphema in patients with proliferative diabetic retinopathy (PDR) who have undergone pars plana vitrectomy (PPV) is rarely documented in the literature. As patients with neovascularization of the iris or angles and neovascular glaucoma (NVG) have a higher risk of hyphema, we investigated the frequency, risks, and treatment regimens associated with hyphema after PPV for patients with PDR without neovascularization of the iris or angles and NVG. In this study, we reviewed the surgical records and postoperative course records of patients with PDR without neovascularization of the iris or angles and NVG after undergoing PPV and identified the patients with postoperative hyphema. We describe the incidence, risk factors, and management protocols of hyphema in these patients. The effect of hyphema on visual recovery and the final anatomical success is also analyzed and described here.

\section{Methods}

We performed a retrospective review of 155 consecutive eyes in 124 patients with PDR without neovascularization of the iris or angles and NVG who had undergone PPV in the Department of Ophthalmology on the East campus of the Renmin Hospital of the Wuhan University (Wuhan Optics Valley General Hospital) from October 2017 to December 2019. The university's ethics committee and institutional review board approved the study protocol, which adhered to the tenets outlined in the Declaration of Helsinki. Informed consent was obtained from each patient before their procedure. The indications for patients with PDR who underwent PPV were vitreous hemorrhage $(\mathrm{VH})$, recurrent $\mathrm{VH}$, massive preretinal hemorrhage, extensive fibrovascular proliferation, tractional retinal detachment, and tractionalrhegmatogenous retinal detachment. Patients with NVG, neovascularization of the iris or angles and ocular trauma were excluded. All surgeries were performed by the same experienced physician (Dr. Zhen Chen). The patients' clinical data, including age, gender, type and duration of diabetes mellitus (DM), coexisting systemic diseases (such as hypertension), use of oral anticoagulant/antiplatelet medications, recent surgical procedures, best corrected visual acuity (BCVA), IOP, slit-lamp biomicroscopy, dilated fundus examination, and fundus photographs (or B-scan ultrasonography) were collected prior to surgery. Postoperative examinations included BCVA, IOP, slit-lamp biomicroscopy, and dilated fundus examination.

BCVA was measured using a Snellen chart, and the values were converted to logarithm of the minimum angle of resolution $(\log M A R)$ scores for data analysis. The visual acuity levels of counting fingers, hand motion, and light perception were recorded as $2.0 \log \mathrm{MAR}, 3.0$ $\log \mathrm{MAR}$, and $3.3 \log \mathrm{MAR}$, respectively.

All cases were performed under either general anesthesia or local retrobulbar anesthesia. Some patients received an intravitreal ranibizumab (Lucentis; Novartis, Switzerland and Genentech, USA; $0.5 \mathrm{mg}$ in $0.05 \mathrm{~mL}$ ) 3-5 days before undergoing vitrectomy. In all cases, a 23gauge vitrectomy (Bausch \& Lomb, St Louis, MO, USA) with a wide-angle viewing system (Alcon Laboratories, Fort Worth, TX, USA) was used. After surgery, all patients were examined daily in the ward to assess BCVA and IOP and to undergo slit-lamp biomicroscopy and dilated fundus examination. All patients underwent PPV alone or in combination with phacoemulsification. During PPV, the cortical vitreous and/or hemorrhage was first removed. This was followed by vitreous base shaving $\left(360^{\circ}\right)$ under scleral depression to remove the peripheral vitreous, and then the fibrovascular membrane was removed using the bimanual or unimanual technique. Scissors segmentation and delamination were used to divide the thick fibrotic plaque from the retina, then fibrovascular membrane was removed by vitreous cutter. The bimanual technique such as membrane dissections and en bloc excision was primarily used in complicated situations during vitrectomy. Intraoperatively, bleeding was controlled by endodiathermy or increasing the infusion or air pressure. After fluid-air exchange, retinal breaks were treated with endolaser, complete panretinal photocoagulation (PRP) was performed on the anterior retina in all cases, and air or silicone oil (SO, Oxane 5700 centistokes, Bausch \& Lomb Inc. Rochester, NY, USA) was used as a tamponade. The sclerotomy sites were routinely sutured with $8 / 0$ polyglactin, and patients were instructed to maintain an appropriate head posture after surgery.

Clinicians generally grade hyphema based on the percentage of the anterior chamber that is filled with blood. Grade 0 (microhyphema) occurs when there are scattered red blood cells in the anterior chamber that do not form 
a layer. Grade I hyphema occurs when $<33 \%$ of the anterior chamber is filled with blood, grade II has 33-50\% filling, grade III has $>50 \%$ but $<100 \%$ filling, and grade IV has $100 \%$ filling. ${ }^{1,13}$

The patients were divided into two groups based on whether they displayed hyphema in the anterior chamber postoperatively. Group 1 included eyes from patients with PDR who did not develop hyphema after PPV, and group 2 included eyes from patients with PDR who developed hyphema after PPV. These two groups were compared to identify and analyze the possible risk factors for postoperative hyphema.

The incidence and management of postoperative hyphema and the time of hemorrhage absorption were recorded. Patients with hyphema were treated with medical agents and/or surgical procedures. Conservative management options included bed rest, head elevation, the use of an eye shield, and the use of pharmacological agents (topical steroids and anti-glaucoma medications). We found that patients with hyphema also displayed a high IOP, which is defined as a pressure $>21 \mathrm{mmHg}$ as measured by non-contact tonometer (FT-1000; Tomey Corporation, Japan). High IOP was controlled using topical $\beta$-adrenergic antagonists, $\alpha$-adrenergic agonists, and topical and oral carbonic anhydrase inhibitors. If no improvement was observed after using these medications, surgical intervention was required. The outcome measures used after absorption of hyphema were visual recovery, IOP, and retinal anatomical success, anatomical success was defined as retinal reattachment during follow up. We also recorded the postoperative complications in the hyphema group.

The preoperative and postoperative BCVA of patients with hyphema were analyzed using a paired $t$-test. Statistical comparison between groups 1 and 2 was performed using a chi-square test, Fisher's test, or MannWhitney $U$-test, and categorical variables included sex, age, duration of DM, hypertension, use of oral anticoagulation agents, preoperative IOP, indications for surgery, anesthesia, preoperative use of intravitreal ranibizumab (IVR), endodiathermy, membrane removal, method of membrane removal, iatrogenic tears, fluid-air exchange, postoperative head position, and surgical time.

We assessed the potential risk factors for postoperative hyphema using mixed-effect logistic regression analysis. A p-value of $<0.05$ was used as the threshold to determine statistical significance. Statistical analyses were performed using SPSS version 17.0 (SPSS Inc., Chicago, IL, USA) and $\mathrm{R}$ language version 3.6.2.

\section{Results}

A total of 155 consecutive eyes from 124 patients with PDR without neovascularization of the iris or angles and NVG who had undergone PPV were enrolled in this retrospective study. Of the 155 procedures, postoperative hyphema occurred in 18 eyes $(11.6 \%)$ after PPV. The hyphema group $(\mathrm{n}=18)$ included 10 males and 8 females, and the non-hyphema group $(\mathrm{n}=106)$ included 60 males and 46 females. Table 1 compares the baseline data and preoperative characteristics between the hyphema and non-hyphema group and shows that there were no significant differences in terms of demographic and baseline data and preoperative characteristics including sex, age, duration of DM, hypertension, use of oral anticoagulants, preoperative IOP, history of PRP, and indications for surgery.

Table I Baseline Data and Preoperative Characteristics of PDR Patients for PPV

\begin{tabular}{|c|c|c|c|}
\hline & $\begin{array}{c}\text { Hyphema } \\
(+) n=18\end{array}$ & $\begin{array}{l}\text { Hyphema } \\
(-) n=106\end{array}$ & $P$ value \\
\hline No. of eyes & 18 & 137 & \\
\hline Sex & & & 1 \\
\hline Male & 10 & 60 & \\
\hline Female & 8 & 46 & \\
\hline Age & & & 0.191 \\
\hline$<40$ & I & 16 & \\
\hline $40-60$ & 12 & 58 & \\
\hline$>60$ & 5 & 32 & \\
\hline Duration of DM & $12.4 \pm 10.6$ & $10.7 \pm 6.6$ & 0.699 \\
\hline Hypertension & 9 & 42 & 0.276 \\
\hline Oral anticoagulants & 1 & 5 & 0.572 \\
\hline Preoperative IOP (mmHG) & $16.6 \pm 3.6$ & $16.0 \pm 2.9$ & 0.431 \\
\hline PRP & 2 & 21 & 0.489 \\
\hline \multicolumn{4}{|l|}{ Indications of the surgery } \\
\hline $\mathrm{VH}$ & 12 & 104 & 1 \\
\hline Recurrent VH & 0 & 6 & 0.798 \\
\hline Massive preretinal hemorrhage & 0 & 2 & 0.067 \\
\hline FVP & 8 & 42 & 0.286 \\
\hline TRD & 6 & 19 & 0.16 \\
\hline TRD/RRD & 0 & 11 & 0.649 \\
\hline
\end{tabular}

Note: $\mathrm{P}$ value was obtained by Chi-square test or Fisher exact test or $t$-test. Abbreviations: DM, diabetes mellitus; IOP, intraocular pressure; PRP, panretinal photocoagulation; VH, vitreous hemorrhage; FVP, fibrovascular proliferation; TRD, tractional retinal detachment; TRD/RRD, tractional-rhegmatogenous retinal detachment. 
The surgical procedures of PPV used for patients with PDR are listed in Table 2. Of the 18 eyes, 7 (38.9\%) received IVR before surgery, and 11 (61.1\%) did not. Intraoperative fibrovascular membrane removal was performed on 15 eyes (83.3\%), 10 (55.6\%) underwent unimanual vitrectomy, 5 (27.8\%) underwent bimanual vitrectomy, $5(27.8 \%)$ received endodiathermy to stop bleeding, 5 (27.8\%) had iatrogenic tears after membrane removal, fluid-air exchange was performed on all eyes, and all were filled with SO after surgery. The surgical time was $80 \pm 15 \mathrm{~min}$ in the hyphema group and $65 \pm$ $20 \mathrm{~min}$ in the non-hyphema group, and statistical analysis showed that the surgical time in the hyphema group was significantly longer than in the non-hyphema group $(\mathrm{p}<$ $0.001)$. In addition, the $t$-test indicated that the surgical time in the membrane removal group was significantly longer than in the non-membrane removal group $(\mathrm{t}=$ $10.7 ; p<0.001)$, which shows that membrane removal increases the surgical time, resulting in the correlation between surgical time and hyphema. Statistical analysis revealed that the two groups did not differ significantly based on anesthesia, IVR pretreatment, endodiathermy, method of membrane removal, iatrogenic tears, fluid-air exchange, or postoperative head position. However, hyphema occurred more frequently in patients with PDR who underwent membrane removal during PPV ( $p=$ 0.021). In our study population, all eyes with postoperative hyphema were filled with SO. Using SO tamponade might influence the results, but we did not analyze the choice of tamponade as an influencing factor. Other postoperative complications after vitrectomy were recorded in the hyphema group. Early postoperative VH within 1 month after the surgery occurred in 3 eyes $(16.7 \%)$, preretinal bleeding occurred in 15 eyes $(66.7 \%)$, recurrent retinal detachment complicated by progressive fibrovascular proliferation developed in 1 eyes $(5.6 \%)$. Later postoperative $\mathrm{VH}$, choroidal detachment, endophthalmitis and NVG were not observed in the hyphema group.

Table 3 shows the clinical features and outcomes of the postoperative hyphema cases in this cohort of patients. Of the 18 eyes, the mean age of the patients was $53.4 \mathrm{y}$, and the mean duration of DM was $12.4 \mathrm{y}$. Of these eyes, 14 had postoperative hyphema from the first postoperative day, and, in 4 cases, postoperative hyphema occurred 7-20 days after surgery. Of the 18 cases of hyphema, $12(66.7 \%)$ were grade I, 2 (11.1\%) were

Table 2 Surgical Procedures of PDR Patients for PPV

\begin{tabular}{|c|c|c|c|}
\hline & Hyphema (+) n= I 8(Eyes) & Hyphema (-) $N=137$ (Eyes) & $\mathbf{P}$ value \\
\hline Anesthesia & & & I \\
\hline General anesthesia & 0 & $\mathrm{I}(0.7 \%)$ & \\
\hline Local retrobulbar anesthesia & $18(100 \%)$ & 136(99.3\%) & \\
\hline IVR pretreatment & $7(38.9 \%)$ & $72(52.6 \%)$ & 0.401 \\
\hline Endodiathermy & $5(27.8 \%)$ & $35(25.5 \%)$ & 0.584 \\
\hline Membrane removal & $15(83.3 \%)$ & $72(52.6 \%)$ & 0.021 \\
\hline Method of removing membrane & & & I \\
\hline No membrane removal & $3(16.7 \%)$ & $65(47.4 \%)$ & \\
\hline Unimanual & $10(55.6 \%)$ & $49(35.8 \%)$ & \\
\hline Bimanual & $5(27.8 \%)$ & $23(16.8 \%)$ & \\
\hline latrogenic tears & $5(27.8 \%)$ & $22(16.1 \%)$ & 0.082 \\
\hline Fluid-air exchange & $18(100 \%)$ & $114(83.2 \%)$ & 0.076 \\
\hline \multicolumn{4}{|l|}{ Tamponade procedure } \\
\hline No tamponade & 0 & $23(16.8 \%)$ & - \\
\hline Air tamponade & 0 & $44(32.1 \%)$ & \\
\hline SO tamponade & $18(100 \%)$ & $70(51.1 \%)$ & \\
\hline Postoperative head position & & & 0.076 \\
\hline Prone & $18(100 \%)$ & $114(83.2 \%)$ & \\
\hline Not prone & 0 & $23(16.8 \%)$ & \\
\hline Surgical time $(\mathrm{min})$ & $80 \pm 15$ & $65 \pm 20$ & $<0.001$ \\
\hline
\end{tabular}

Note: $\mathrm{P}$ value was obtained by $\mathrm{Chi}$-square test or Fisher exact test or $t$-test.

Abbreviations: IVR, intravitreal ranibizumab; SO, silicone oil. 


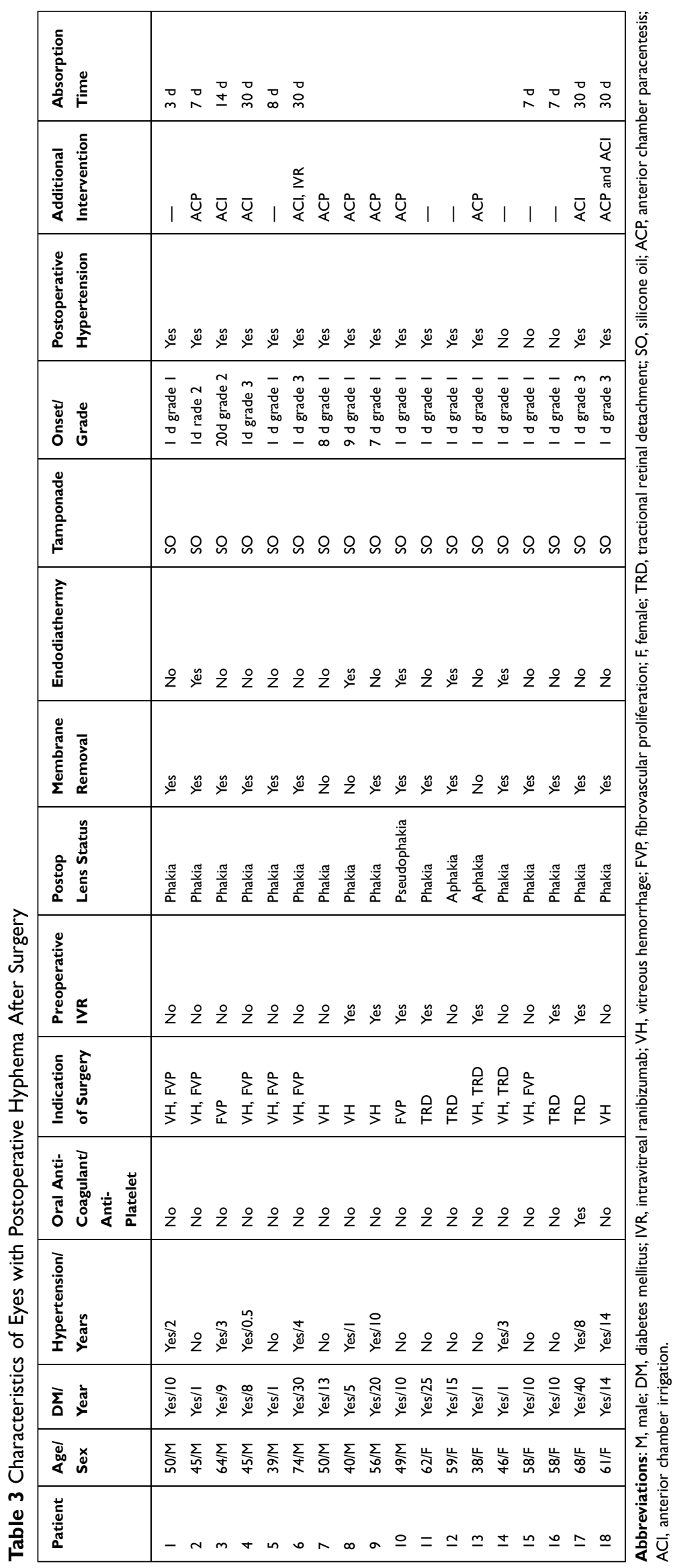


grade II, 4 (22.2\%) were grade III, 3 (16.7\%) had normal IOP, and $15(83.3 \%)$ had elevated IOP. Of the patients with high IOP, only 4 cases (22.2\%) were normalized using IOP-lowering drugs alone. In addition to using IOPlowering drugs, six patients $(33.3 \%)$ required anterior chamber paracentesis (ACP), one (5.6\%) required anterior chamber irrigation (ACI) combined with ACP, four (22.2\%) required only $\mathrm{ACI}$, and one $(5.6 \%)$ required $\mathrm{ACI}$ combined with IVR. We performed ACP as an outpatient procedure using a slit lamp. IOP was controlled by topical medications, ACP, or ACI in all patients with hyphema, except for one eye that required a glaucoma valve implantation. The average time for absorption of the hyphema was $13.1 \mathrm{~d}(3-30 \mathrm{~d})$. No recurrence of hyphema was observed.

During follow-up examinations, we found that all 18 eyes with hyphema achieved anatomical success, the final anatomic success rate was $100 \%$, and the final BCVA for each patient improved significantly when compared with their initial BCVA. The mean postoperative BCVA was $1.75 \pm 0.84 \log$ MAR, which is a significant improvement over the preoperative BCVA of $2.20 \pm 0.65 \operatorname{logMAR}(\mathrm{t}=$ 3.893; $\mathrm{p}=0.001)$.

We also analyzed the potential independent risk factors for the development of hyphema after PDR surgeries using mixed-effect logistic regression. The results indicated that membrane removal was a significant risk factor for the development of hyphema (OR $=5.65$ and $95 \%$ CI: 1.190 25.203; $\mathrm{p}=0.013)$.

\section{Discussion}

Diabetic retinopathy (DR) is the most common microvascular complication of DM and is responsible for up to $4.8 \%$ of cases of blindness globally. ${ }^{14,15}$ If left untreated, nearly half of the eyes that develop PDR will also develop severe vision loss due to related complications, including retinal detachment and vitreous hemorrhage. ${ }^{16}$ Over the past decade, rapid advancements in technology for retinal imaging and the development of new therapies have dramatically improved the visual outcomes for patients with DR. ${ }^{16}$ In cases of severe PDR, such as those with a dense and extensive fibrovascular membrane and tractional retinal detachment, vitreoretinal surgery is necessary, which can restore vision and improve the patient's quality of life.

However, this procedure can be associated with complications, including recurrent vitreous hemorrhage, retinal detachment, cataract, neovascular glaucoma, and endophthalmitis. ${ }^{17}$ Although rare, hyphema can develop in certain cases after PPV for PDR. ${ }^{18-20}$ From the literature, it is clear that postoperative hyphema is a frequent complication of trabeculectomy in patients with NVG, and hyphema may be a surgical risk factor for the failure of trabeculectomy in these patients. In this group, the preoperative use of intravitreal bevacizumab (IVB) can reduce the risk of postoperative hyphema. ${ }^{4,21-23}$ In our study, there were no patients with neovascularization of the iris or angles and NVG in the hyphema or the non-hyphema group, and we believe that the mechanism of hyphema in patients with NVG is different from its mechanism in the group of patients we studied.

Among the hemorrhagic complications that can occur after PPV for PDR without neovascularization of the iris or angles and NVG, postoperative hyphema is relatively rare when compared with the development of a vitreous hemorrhage. Hyphema often develops from tears in the vessels of the ciliary body and iris following trauma or an iridectomy. ${ }^{3}$ In the present study, $11.6 \%$ of patients with PDR developed postoperative hyphema after undergoing PPV, and $2.3 \%$ (4 of 155 eyes) developed severe hyphema. However, other studies have observed occurrence rates of postoperative hyphema of $5.6 \%,{ }^{19} 10 \%, 16.2 \%,{ }^{18}$ and $8 \%{ }^{20}$ Patients with postoperative hyphema may be asymptomatic, may present with a transient vision blockage, or may associate their symptoms with vitreous hemorrhage. Hyphema does not typically cause permanent loss of vision, ${ }^{24}$ but in some cases, it will inhibit the drainage of aqueous humor resulting in an acute rise in the IOP and optic neuropathy, which ultimately poses a serious threat to the patient's vision. ${ }^{13}$ Of our cohort, 15 eyes (83.3\%) exhibited elevated IOP. The management of hyphema seeks to accelerate the absorption of the blood and prevent possible complications. ${ }^{13}$ Conservative management options included bed rest, head elevation, the use of an eye shield, and the use of pharmacological agents, eg, topical steroids and anti-glaucoma drugs. If these methods failed and clinical indications for surgical treatment were met, including persistent elevated IOP, possible corneal blood staining, and non-resolving hyphema, surgery was performed, and the technique chosen was based on the severity and density of hyphema. ${ }^{13}$ In our study, 4 eyes (22.2\%) were stabilized using only IOP-lowering drugs and 11 required ACP and/or ACI. ACP is the most common outpatient surgical approach, as it can be performed safely and repeatedly using a slit lamp with adequate aseptic precautions. ${ }^{25}$ If there is a small amount of hyphema or large blood cells in the anterior chamber, 
ACP can be used. However, if the blood clots in the anterior chamber are not easily absorbed, ACI should be performed. Nearly all patients with elevated IOP were stabilized using topical medications, ACP, and/or ACI, and only one eye required a glaucoma valve implantation. No recurrence of hyphema was observed.

In all patients who developed postoperative hyphema in our study, SO was used for long-term tamponade to prevent recurrent retinal detachment, improve postoperative visual acuity, reduce the possibility of recurrent bleeding, and complement follow-up laser therapy. In three patients without fibrovascular membrane, SO was used because adequate hemostasis was not achieved at the end of the vitrectomy. Through the surgical records, we found that four patients with severe hyphema developed active bleeding after membrane removal during the surgery. Complete hemostasis could not be achieved by perfusion/ air pressure increase or adequate endodiathermy. When using logistic regression analysis to study the potential risk factors in patients with PDR for developing postoperative hyphema after PPV, we found that membrane removal was a significant independent risk factor $(\mathrm{OR}=5.65$ and 95\% CI: 1.190-25.203; $\mathrm{p}=0.013$ ). As membrane removal was strongly correlated with surgical time, it was not used in the regression model. Membrane dissection is an effective and important technique in the treatment of complex PDR, and bleeding can easily occur when performing this surgery. ${ }^{26}$ In addition, we discovered that most cases of hyphema that develop following intraocular surgery occur at the time of surgery or immediately thereafter. In this study, $83.3 \%$ of patients (15 eyes) that developed hyphema had undergone fibrovascular membrane removal. In addition, 14 eyes developed postoperative hyphema on the first day after surgery, and 4 cases developed hyphema 7-20 days after surgery. We postulate that early hyphema may be caused by blood that is able to break through the silicone oil and pass through the weakened zonules in pseudophakic or phakic eyes into the anterior chamber. However, late hyphema may be caused by rebleeding due to the dispersion of residual blood in the retina, or because the preretinal blood clot liquefaction time was 1-3 weeks (average $14 \mathrm{~d}$ ). ${ }^{27,28}$ Remaining in the prone position after vitrectomy can easily cause the blood clot to dissolve into the anterior chamber. The liquefaction of preretinal bleeding can also cause the hyphema to undergo changes, which might require multiple ACPs and ACIs. Nonetheless, hyphema in the anterior chamber did not ultimately affect the patient's vision or anatomical success.
Aside from membrane removal, we did not find any factors that were associated with the occurrence of hyphema including sex, age, duration of DM, hypertension, use of anticoagulant/antiplatelet medications, preoperative IOP, indications for surgery, anesthesia, preoperative IVR, endodiathermy, method of membrane removal, iatrogenic tears, fluid-air exchange, or postoperative head position. Previous studies have indicated that anticoagulants or antiplatelet agents do not increase the risk of ocular hemorrhagic events in cataract and macular surgery, ${ }^{29-31}$ which is consistent with the results presented here. Some studies have indicated that preoperative IVB can reduce the risk of postoperative hyphema in patients with NVG and patients with PDR. ${ }^{19,23}$ However, IVR did not decrease the occurrence of postoperative hyphema in our study. We speculate that this is because only $38.9 \%$ of patients with hyphema received IVR, and the hyphema group may include more complicated patients with PDR with a high risk of postoperative bleeding.

While our work revealed important insights into the risks that can lead to hyphema, there are some potential limitations. First, it was a retrospective study. Second, the patients with PDR in our study were complex and may not accurately reflect all patients. Despite these limitations, we identified membrane removal as a risk factor for developing postoperative hyphema after PPV in patients with PDR. The incidence of postoperative hyphema was $11.6 \%$, and neither the presence nor the degree of hyphema affected the visual recovery or final anatomical success of the patients. Our results indicate that surgeons should carefully remove the membrane in patients with complex PDR to reduce intraoperative and postoperative bleeding. However, if hyphema develops postoperatively, timely treatment will reduce complications and allow for complete recovery.

\section{Disclosure}

The authors report no conflicts of interest in this work.

\section{References}

1. Brandt MT, Haug RH. Traumatic hyphema: a comprehensive review. J Oral Maxillofac Surg. 2001;59(12):1462-1470. doi:10.1053/ joms.2001.28284

2. Iftikhar M, Mir T, Seidel N, et al. Epidemiology and outcomes of hyphema: a single tertiary centre experience of 180 cases. Acta Ophthalmol. 2021;99(3):e394-e401. doi:10.1111/aos.14603

3. Sankar PS, Chen TC, Grosskreutz CL, Pasquale LR. Traumatic hyphema. Int Ophthalmol Clin. 2002;42(3):57-68. doi:10.1097/ 00004397-200207000-00008 
4. Alkawas AA, Shahien EA, Hussein AM. Management of neovascular glaucoma with panretinal photocoagulation, intravitreal bevacizumab, and subsequent trabeculectomy with mitomycin C. J Glaucoma. 2010;19(9):622-626. doi:10.1097/IJG.0b013e $3181 \mathrm{ccb} 794$

5. Hong JW, Choi GJ. Ahmed valve implantation for refractory glaucoma following pars plana vitrectomy. Korean J Ophthalmol. 2005;19(4):293-296. doi:10.3341/kjo.2005.19.4.293

6. Buffault J, Graber M, Bensmail D, et al. Efficacy and safety at 6 months of the XEN implant for the management of open angle glaucoma. Sci Rep. 2020;10(1):4527. doi:10.1038/s41598-02061319-1

7. Zhang Q, Zhao M, Xu M, et al. Outcomes of therapeutic keratoplasty for severe infectious keratitis in Chongqing, a 16-year experience. Infect Drug Resist. 2019;12:2487-2493. doi:10.2147/IDR.S204025

8. Sharma U, Sharma B, Kumar K, Kumar S. Evaluation of complications and visual outcome in various nucleus delivery techniques of manual small incision cataract surgery. Indian J Ophthalmol. 2019;67 (7):1073-1078. doi:10.4103/ijo.IJO_1261_18

9. Zhuang M, Fan W, Xie P, Yuan ST, Liu QH, Zhao C. Evaluation of the safety and quality of day-case cataract surgery based on 4151 cases. Int J Ophthalmol. 2019;12(2):291-295.

10. Hasan SU, Rizvi SF, Mahmood SA, Zafar S, Hasan T. Anatomical outcome following indocyanine green assisted internal limiting membrane peeling for stage 3 and 4 macular hole surgery. $J$ Coll Physicians Surg Pak. 2017;27(8):486-489.

11. Sayed KM, Naito T, Farouk MM, et al. Twenty five-gauge sutureless vitrectomy versus 20-gauge vitrectomy in epiretinal membrane surgery. J Med Invest. 2012;59(1-2):69-78. doi:10.2152/jmi.59.69

12. Kung Y, Wu T. Risk factors of hyphema following surgeries for primary rhegmatogenous retinal detachment. Ophthalmologica. 2016;236(3):159-165. doi:10.1159/000452254

13. Bansal SMM, Gunasekeran DVM, Ang BM, et al. Controversies in the pathophysiology and management of hyphema. Surv Ophthalmol. 2016;61(3):297-308. doi:10.1016/j.survophthal.2015.11.005

14. Resnikoff S, Pascolini D, Etya'Ale D, et al. Global data on visual impairment in the year 2002. Bull World Health Organ. 2004;82 (11):844-851.

15. Fong DS, Aiello LP, Ferris FR, Klein R. Diabetic retinopathy. Diabetes Care. 2004;27(10):2540-2553. doi:10.2337/ diacare.27.10.2540

16. Jampol LM, Glassman AR, Sun J. Evaluation and care of patients with diabetic retinopathy. $N$ Engl J Med. 2020;382(17):1629-1637. doi:10.1056/NEJMra1909637

17. Yau GL, Silva PS, Arrigg PG, Sun JK. Postoperative complications of pars plana vitrectomy for diabetic retinal disease. Semin Ophthalmol. 2018;33(1):126-133.

18. Chung TY, Chung H, Lee JH. Combined surgery and sequential surgery comprising phacoemulsification, pars plana vitrectomy, and intraocular lens implantation: comparison of clinical outcomes. J Cataract Refract Surg. 2002;28(11):2001-2005. doi:10.1016/ S0886-3350(02)01354-8
19. Zaman Y, Rehman AU, Memon AF. Intravitreal Avastin as an adjunct in patients with proliferative diabetic retinopathy undergoing pars plana vitrectomy. Pak J Med Sci. 2013;29(2):590-592. doi:10.12669/pjms.292.3044

20. Oshima Y, Shima C, Wakabayashi T, et al. Microincision vitrectomy surgery and intravitreal bevacizumab as a surgical adjunct to treat diabetic traction retinal detachment. Ophthalmology. 2009;116 (5):927-938. doi:10.1016/j.ophtha.2008.11.005

21. Nakatake S, Yoshida S, Nakao S, et al. Hyphema is a risk factor for failure of trabeculectomy in neovascular glaucoma: a retrospective analysis. BMC Ophthalmol. 2014;14:55. doi:10.1186/1471-2415-14-55

22. Kojima S, Inatani M, Shobayashi K, Haga A, Inoue T, Tanihara H. Risk factors for hyphema after trabeculectomy with mitomycin C. J Glaucoma. 2014;23(5):307-311. doi:10.1097/IJG.0b013e3 $182741 \mathrm{c} 85$

23. Saito Y, Higashide T, Takeda H, Ohkubo S, Sugiyama K. Beneficial effects of preoperative intravitreal bevacizumab on trabeculectomy outcomes in neovascular glaucoma. Acta Ophthalmol. 2010;88 (1):96-102. doi:10.1111/j.1755-3768.2009.01648.x

24. Gharaibeh A, Savage HI, Scherer RW, Goldberg MF, Lindsley K. Medical interventions for traumatic hyphema. Cochrane Database Syst Rev. 2019;1:D5431.

25. Trivedi D, Denniston AK, Murray PI. Safety profile of anterior chamber paracentesis performed at the slit lamp. Clin Exp Ophthalmol. 2011;39(8):725-728. doi:10.1111/j.1442-9071.20 11.02565.x

26. Newman DK. Surgical management of the late complications of proliferative diabetic retinopathy. Eye. 2010;24(3):441-449. doi:10.1038/eye.2009.325

27. Quiroz-Mercado H, Garza-Karren CD, Roigmelo EA, JimenezSierra JM, Dalma-Weiszhausz J. Vitreous management in massive suprachoroidal hemorrhage. Eur J Ophthalmol. 1997;7(1):101-104. doi:10.1177/112067219700700117

28. Lakhanpal V, Schocket SS, Elman MJ, Nirankari VS. A new modified vitreoretinal surgical approach in the management of massive suprachoroidal hemorrhage. Ophthalmology. 1989;96(6):793-800. doi:10.1016/S0161-6420(89)32819-3

29. Katz J, Feldman MA, Bass EB, et al. Risks and benefits of anticoagulant and antiplatelet medication use before cataract surgery. Ophthalmology. 2003;110(9):1784-1788. doi:10.1016/S01616420(03)00785-1

30. Li Q, Shen X, Wang S, et al. Combined aspirin and clopidogrel therapy in phacoemulsification cataract surgery: a risk factor for ocular hemorrhage? Int Ophthalmol. 2020;40:2023-2029.

31. Louison S, Gabrielle PH, Soudry A, et al. Perioperative risk of bleeding with antithrombotic agents in macular surgery: a national, prospective, multicentre study. Acta Ophthalmol. 2020;98. doi:10.1111/aos.14434
International Journal of General Medicine

\section{Publish your work in this journal}

The International Journal of General Medicine is an international, peer-reviewed open-access journal that focuses on general and internal medicine, pathogenesis, epidemiology, diagnosis, monitoring and treatment protocols. The journal is characterized by the rapid reporting of reviews, original research and clinical studies across all disease areas. The manuscript management system is completely online and includes a very quick and fair peer-review system, which is all easy to use. Visit http://www.dovepress.com/ testimonials.php to read real quotes from published authors. 